Bull. Austral. Math. Soc.

Vol. 63 (2001) [1-14]

\title{
AN EXISTENCE THEOREM FOR NONLINEAR HEMIVARIATIONAL INEQUALITIES AT RESONANCE
}

\author{
Leszek Gasiński and Nikolaos S. Papageorgiou
}

\begin{abstract}
We consider a nonlinear hemivariational inequality with the p-Laplacian at resonance. Using an extension of the nonsmooth mountain pass theorem of Chang, which makes use of the Cerami compactness condition, we prove the excistence of a nontrivial solution. Our existence results here extends a recent theorem on resonant hemivariational inequalities, by the authors in 1999 .
\end{abstract}

\section{INTRODUCTION}

In a recent paper (see Gasinski and Papageorgiou [9]), we examined a nonlinear hemivariational inequality at resonance. We proved an existence theorem under the assumptions that the Clarke subdifferential of the generalised potential is bounded and that it has nonzero limits at $\pm \infty$. In the present paper, we remove both these restrictive hypotheses and instead we assume a general $(p-1)$-growth condition for the subdifferential and a behaviour in the "neighbourhood of $\pm \infty$ ", which is a variation of the well known Ambrosetti-Rabinowitz condition (see [3]).

Semilinear (that is, for $p=2$ ) hemivariational inequalities at resonance were studied by Goeleven, Motreanu and Panagiotopoulos in [12]. While our hypotheses do not preclude the situation where $\lim _{|t| \rightarrow+\infty} \int_{Z} j\left(z, t u_{1}(z)\right) d z$ may be finite $\left(u_{1}\right.$ being the principal eigenfunction of the $p$-Laplacian), in [12], when the problem is formulated in our setting with $p=2$ (that is, resonance at the principal eigenvalue $\lambda_{1}$ ), the authors always require that $\lim _{|t| \rightarrow+\infty} \int_{Z} j\left(z, t u_{1}(z)\right) d z$ is infinite. Moreover, their growth conditions on the subdifferential $\partial j(z, \zeta)$ are more restrictive and in [12, Section 5], they assume that the Clarke subdifferential of $J(x)=\int_{Z} j(z, x(z)) d z$ admits a continuous selector $v: L^{2}(Z) \mapsto L^{2}(Z)$. This is a rather severe restriction if we take into consideration the fact that in general the Clarke subdifferential of a locally Lipschitz function is only upper semicontinuous from the Banach space into its dual furnished with the $w^{*}$-topology (see Section 2). Other (nonresonant) eigenvalue problems for hemivariational inequalities were studied recently by Goeleven, Motreanu and Panagiotopoulos [11] and Gasiński and Papageorgiou [10].

Received 2nd December, 1999

Copyright Clearance Centre, Inc. Serial-fee code: 0004-9727/01 \$A2.00+0.00. 
Hemivariational inequalities arise in physical and engineering problems, where the consideration of more realistic laws leads to nonconvex, nonsmooth energy functionals and to a new type of variational expression, namely the hemivariational inequality. For such a model the eigenvalue problem is closely connected with the stability analysis of the corresponding mechanical system. So for instance in beam buckling theory, the lower eigenvalue corresponds to the critical loading, that is, to the maximum loading sustained by the structure before instability occurs. Additional mechanical applications, such as the study of adhesive joints in structural mechanics, the behaviour of composites, noncovex semipermeability, unilateral contact and nonmonotone problems are studied in the recent book of Panagiotopoulos [17].

Also we should mention that hemivariational inequalities include, as a special case, problems with discontinuities. These correspond to the case when the generalised potential function is of the form $j(z, \zeta)=\int_{0}^{\zeta} f(z, \xi) d \xi$, with $f$ being a Borel measurable function, in general discontinuous in the second variable. Problems with discontinuities were studied by Chang in [7], where, for this purpose, a nonsmooth extension of the classical critical point theory is developed. Previous works for resonant elliptic problems deal with the semilinear case and of course assume that $j(z, \zeta)=\int_{0}^{\zeta} f(z, \xi) d \xi$, with $f \in C(Z \times \mathbb{R})$ (hence $j \in C^{1}(Z \times \mathbb{R})$ ). We refer to the works of Ahmad, Lazer and Paul [2] (where $j(z, \zeta) \rightarrow+\infty$ as $\zeta \rightarrow \pm \infty$ ), Bartolo, Benci and Fortunato [4], Thew [20] and Ward [22] (where $j(z, \zeta)$ has finite limits at $\pm \infty$; this is the so called strong resonant problem).

\section{Preliminaries}

Our approach is variational and uses the critical point theory of Chang [7] for nonsmooth locally Lipschitz functionals. The work of Chang was based on the subdifferential theory of Clarke [8]. In this section, for the convenience of the reader, we recall some basic definitions and facts from these theories, which we shall use in the sequel.

Let $X$ be a Banach space and $X^{*}$ its topological dual. By $\|\cdot\|$ we shall denote the norm in $X$, by $\|\cdot\|_{*}$ the norm in $X^{*}$, and by $\langle\cdot, \cdot\rangle$ the duality brackets for the pair $\left(X, X^{*}\right)$. A function $\phi: X \mapsto \mathbb{R}$ is said to be locally Lipschitz, if for every $x \in X$ there exists a neighbourhood $U$ of $x$ and a constant $k>0$ depending on $U$ such that $|\phi(x)-\phi(y)| \leqslant k\|z-y\|$ for all $z, y \in U$. From convex analysis it is known that a proper, convex and lower semicontinuous function $g: X \mapsto \overline{\mathbf{R}} \stackrel{d f}{=} \mathbb{R} \cup\{+\infty\}$ is locally Lipschitz in the interior of its effective domain $\operatorname{dom} g \stackrel{d f}{=}\{x \in X: g(x)<+\infty\}$. In analogy with the directional derivative of a convex function, we define the generalised directional derivative of a locally Lipschitz function $\phi$ at $x \in X$ in the direction $h \in X$, 
by

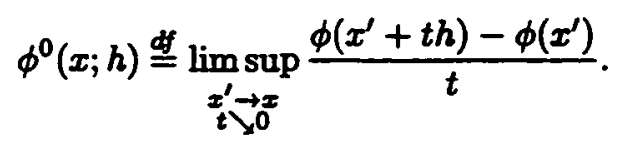

The function $X \ni h \mapsto \phi^{0}(x ; h) \in \mathbf{R}$ is sublinear, continuous and by the Hahn-Banach Theorem it is the support function of a nonempty, convex and $w^{*}$-compact set

$$
\partial \phi(x) \stackrel{d f}{=}\left\{x^{*} \in X^{*}:\left\langle x^{*}, h\right\rangle \leqslant \phi^{0}(x ; h) \text { for all } h \in X\right\} .
$$

This set is called the "generalised" or "Clarke" subdifferential of $\phi$ at $x$. If $\phi, \psi: X \mapsto \mathbf{R}$ are locally Lipschitz functions, then $\partial(\phi+\psi)(x) \subseteq \partial \phi(x)+\partial \psi(x)$ and $\partial(t \phi)(x)=$ $t \partial \phi(x)$ for all $t \in \mathbb{R}$. Moreover, if $\phi: X \mapsto \mathbf{R}$ is also convex, then the subdifferential of $\phi$ in the sense of convex analysis coincides with the generalised subdifferential introduced above. If $\phi$ is strictly differentiable at $x$ (in particular if $\phi$ is continuously Gateaux differentiable at $x)$, then $\partial \phi(x)=\left\{\phi^{\prime}(x)\right\}$.

Let $\phi: X \mapsto \mathbb{R}$ be a locally Lipschitz function on a Banach space $X$. A point $x \in X$ is said to be a "critical point" of $\phi$, if $0 \in \partial \phi(x)$. If $x \in X$ is a critical point of $\phi$, then the value $c \stackrel{d f}{=} \phi(x)$ is called a "critical value" of $\phi$. It is easy to see that, if $x \in X$ is a local extremum of $\phi$, then $0 \in \partial \phi(x)$. Moreover, the multifunction $X \ni x \mapsto \partial \phi(x) \in 2^{X^{*}}$ is upper semicontinuous, where the space $X^{*}$ is equipped with the $w^{*}$-topology, that is, for any $w^{*}$-open set $U \subseteq X^{*}$, the set $\{x \in X: \partial \phi(x) \subseteq U\}$ is open in $X$ (see Hu and Papageorgiou [13]). For more details on the generalised subdifferential we refer to Clarke [8].

The critical point theory for smooth functions uses a compactness condition known as "the Palais-Smale condition". In our present nonsmooth setting, the condition takes the following form:

A locally Lipschitz function $\phi: X \mapsto \mathbf{R}$ satisfies the "nonsmooth Palais-Smale condition", if any sequence $\left\{x_{n}\right\}_{n \geqslant 1} \subseteq X$ such that $\left\{\phi\left(x_{n}\right)\right\}_{n \geqslant 1}$ is bounded and $m\left(x_{n}\right) \stackrel{d f}{=} \min \left\{\left\|x^{*}\right\|_{*}: x^{*} \in \partial \phi\left(x_{n}\right)\right\} \rightarrow 0$ as $n \rightarrow+\infty$, has a strongly convergent subsequence.

If $\phi \in \mathcal{C}^{1}(X)$, then since $\partial \phi\left(x_{n}\right)=\left\{\phi^{\prime}\left(x_{n}\right)\right\}$, we see that the above defintion of the Palais-Smale condition coincides with the classical one (see Rabinowitz [18]).

A weaker form of the Palais-Smale condition was introduced in the context of the smooth theory by Cerami [6]. In our nonsmooth setting this condition takes the following form:

A locally Lipschitz function $\phi: X \mapsto \mathbf{R}$ satisfies the "nonsmooth Cerami condition", if for any sequence $\left\{x_{n}\right\}_{n \geqslant 1} \subseteq X$ such that the sequence of values 
$\left\{\phi\left(x_{n}\right)\right\}_{n \geqslant 1}$ is bounded and $\left(1+\left\|x_{n}\right\|\right) m\left(x_{n}\right) \rightarrow 0$ as $n \rightarrow+\infty$, there exists a strongly convergent subsequence.

It was proved in the smooth case by Bartolo, Benci and Fortunato (see [4, Theorem 1.3]) that this weaker condition suffices for a deformation theorem and from that, one derives minimax principles. The same can be done in the nonsmooth case, by appropriate modification of the proof of Bartolo, Benci and Fortunato [4] using Chang [7, Lemmata 3.1 up to 3.4] in order to obtain the deformation theorem or by using a recent generalisation of the Ekeland variational principle due to Zhong [23] (see Kourogenis and Papageorgiou [14]). So we can state the following slight generalisation of the nonsmooth mountain pass theorem, due to Chang [7]. (See also Ambrosetti and Rabinowitz [3] for the original smooth version of the theorem.)

THEOREM 1. If

(i) $X$ is a reflexive Banach space and $\phi: X \mapsto \mathbb{R}$ is locally Lipschitz functional which satisfies the nonsmooth Cerami condition;

(ii) there exist real number $r>0$ and two points $x_{1}, x_{2} \in X$ such that $\left\|x_{2}-x_{1}\right\|>r$ and $\max \left\{\phi\left(x_{1}\right), \phi\left(x_{2}\right)\right\}<\inf \left\{\phi(x):\left\|x-x_{1}\right\|=r\right\}$;

(iii) $c$ is defined by

$$
c \stackrel{d f}{=} \inf _{\gamma \in \Gamma} \max _{0 \leqslant t \leqslant 1}\{\phi(\gamma(t))\},
$$

where

$$
\Gamma \stackrel{d f}{=}\left\{\gamma \in \mathcal{C}([0,1], X): \gamma(0)=x_{1}, \gamma(1)=x_{2}\right\},
$$

then

(a) $c$ is a critical value of $\phi$;

(b) $c \geqslant \inf \left\{\phi(x):\left\|x-x_{1}\right\|-r\right\}$;

(c) if additionally $c=\inf \left\{\phi(x):\left\|x-x_{1}\right\|=r\right\}$, then there exists a critical point $x_{0} \in X$ of $\phi$ such that $\phi\left(x_{0}\right)=c$ and $\left\|x_{0}-x_{1}\right\|=r$.

In our hypotheses we shall use the first eigenvalue $\lambda_{1}$ of the negative $p$-Laplacian $-\Delta_{p} x=-\operatorname{div}\left(\|\nabla x\|^{p-2} \nabla x\right)$ with Dirichlet boundary conditions (that is, of $\left(-\Delta_{p}\right.$, $\left.W_{0}^{1, p}(Z)\right)$ ). This is defined as follows. Let $Z \subseteq \mathbb{R}^{N}$ be a bounded domain with boundary $\Gamma$ and let us consider the following eigenvalue problem:

$$
\left\{\begin{array}{l}
-\operatorname{div}\left(\|\nabla x(z)\|_{\mathrm{R}^{N}}^{p-2} \nabla x(z)\right)=\lambda|x(z)|^{p-2} x(z) \text { almost everywhere on } Z \\
\left.x\right|_{\Gamma}=0 .
\end{array}\right.
$$

The least real number $\lambda$ for which (EP) has a nontrivial solution is called the first eigenvalue $\lambda_{1}$ of $\left(-\Delta_{p}, W_{0}^{1, p}(Z)\right)$. This first eigenvalue $\lambda_{1}$ is positive, isolated and 
simple (that is, the associated eigenfunctions are constant multiples of each other). Moreover, we have a variational characterisation of $\lambda_{1}$ via the Rayleigh quotient, that is

$$
\lambda_{1}=\min \left\{\frac{\|\nabla x\|_{p}^{p}}{\|x\|_{p}^{p}}: x \in W_{0}^{1, p}(Z), x \neq 0\right\}
$$

This minimum is realised at the normalised eigenfunction $u_{1}$. Note that if $u_{1}$ minimises the Rayleigh quotient, then so does $\left|u_{1}\right|$ and so we infer that the first eigenfunction $u_{1}$ does not change sign on $Z$. In fact we can show that $u_{1}(z) \neq 0$ almost everywhere on $Z$ and so we can assume that $u_{1}>0$ almost everywhere on $Z$. Moreover by the nonsmooth elliptic regularity theorem of Tolksdorf [21], we have that $u_{1} \in C_{\text {loc }}^{1, \beta}(Z)$ with $0<\beta<1$. For details on the first eigenvalue we refer to Lindqvist [16].

The Lusternik-Schnirelmann theory gives, in addition to $\lambda_{1}$, a whole strictly increasing sequence of positive numbers $\left\{\lambda_{n}\right\}_{n \geqslant 1}$, for which there exist nontrivial solutions of (EP). In other words, the spectrum $\sigma(p)$ of $\left(-\Delta_{p}, W_{0}^{1, p}(Z)\right)$ contains at least these points. For $p \neq 2$ nothing is known in general about the possible existence of other points in $\sigma(p) \subseteq\left[\lambda_{1},+\infty\right)$. If $p=2$ (linear case), the Lusternik-Schnirelmann eigenvalues are the only eigenvalues.

\section{HEMIVARIATIONAL INEQUALITIES AT RESONANCE}

Let $Z \subseteq \mathbb{R}^{N}$ be a bounded domain with $C^{1}$-boundary $\Gamma$ and let $2 \leqslant p<+\infty$. We consider the following quasilinear hemivariational inequality at resonance:

(HVI)

$$
\left\{\begin{array}{l}
-\operatorname{div}\left(\|\nabla x(z)\|_{\mathbb{R}_{N}}^{p-2} \nabla x(z)\right)-\lambda_{1}|x(z)|^{p-2} x(z) \\
\quad \in \partial j(z, x(z)) \text { almost everywhere on } Z \\
\left.x\right|_{\Gamma}=0 .
\end{array}\right.
$$

Our hypotheses on the generalised potential function $j(z, \zeta)$ are the following.

$\mathrm{H}(\mathrm{j})$

$$
j: Z \times \mathbb{R} \mapsto \mathbb{R}
$$

is a function such that:

(i) for all $\zeta \in \mathbb{R}, Z \ni \mapsto j(z, \zeta) \in \mathbb{R}$ is measurable;

(ii) for almost all $z \in Z, \mathbf{R} \ni \zeta \mapsto j(z, \zeta) \in \mathbb{R}$ is locally Lipschtiz;

(iii) for almost all $z \in Z$, all $\zeta \in \mathbb{R}$ and all $\eta \in \partial j(z, \zeta)$ we have $|\eta| \leqslant$ $a(z)+c|\zeta|^{p-1}$ with some $a \in L^{\infty}(Z)$ and $c>0$;

(iv) there exist $\beta>0$ and $0<\mu<p$ such that

$$
\liminf _{|\zeta| \mapsto+\infty} \frac{u^{*}(z, \zeta) \zeta-p j(z, \zeta)}{|\zeta|^{\mu}}>\beta
$$


or

$$
\limsup _{|\zeta| \rightarrow+\infty} \frac{u^{*}(z, \zeta) \zeta-p j(z, \zeta)}{|\zeta|^{\mu}}<-\beta,
$$

uniformly for almost all $z \in Z$ and all $u^{*}(z, \zeta) \in \partial j(z, \zeta)$, with $u^{*}(\cdot, \zeta) \in$ $L^{2}(Z)$;

(v) $\lim _{\zeta \rightarrow 0}\left(p j(z, \zeta) /|\zeta|^{p}\right) \leqslant-\lambda_{1}$ uniformly for almost all $z \in Z$;

(vi) $j(\cdot, 0) \in L^{\infty}(Z), \int_{z} j(z, 0) d z \geqslant 0$ and there exists $\xi \neq 0$ such that $\int_{Z} j\left(z, \xi u_{1}(z)\right) d z \geqslant 0$.

Let $\phi: W_{0}^{1, p}(Z) \mapsto \mathbb{R}$ be the energy functional defined by

$$
\phi(x) \stackrel{d f}{=} \frac{1}{p}\|\nabla x\|_{p}^{p}-\frac{\lambda_{1}}{p}\|x\|_{p}^{p}-\int_{z} j(z, x(z)) d z .
$$

Let $\phi: W_{0}^{1, p}(Z) \mapsto \mathbb{R}$ be defined by $\psi(x) \stackrel{d f}{=} \int_{Z} j(z, x(z)) d z$. By virtue of hypothesis $H(j)$ (iii) and Clarke [8, Theorem 2.7.5, p.83], we see that $\psi$ is locally Lipschitz. Also the functionals $W_{0}^{1, p}(Z) \ni x \mapsto\|\nabla x\|_{p}^{p} \in \mathbb{R}$ and $W_{0}^{1, p}(Z) \ni x \mapsto\|x\|_{p}^{p} \in \mathbb{R}$ are convex, continuous, hence locally Lipschitz on $W_{0}^{1, p}(Z)$. Therefore $\phi$ is locally Lipschitz.

LEMMA 2. If hypotheses $H(j)$ hold, then $\phi$ satisfies the nonsmooth Cerami condition.

Proof: We assume that in hypothesis $H(j)$ (iv) the first alternative holds. The proof is similar if the second alternative is in effect.

Let $\left\{x_{n}\right\}_{n \geqslant 1} \subseteq W_{0}^{1, p}(Z)$ be a sequence such that $\left|\phi\left(x_{n}\right)\right| \leqslant M_{1}$ for all $n \geqslant 1$ and $\left(1+\left\|x_{n}\right\|\right) m\left(z_{n}\right) \rightarrow 0$ and $n \rightarrow+\infty$.

Let $x_{n}^{*} \in \partial \phi\left(x_{n}\right)$ be such that $m\left(x_{n}\right)=\left\|x_{n}^{*}\right\|_{*}$, for $n \geqslant 1$. For every $n \geqslant 1$, its existence is a consequence of the fact that $\partial \phi\left(x_{n}\right) \subseteq\left(W_{0}^{1, p}(Z)\right)^{*}=W^{-1, p^{\prime}}(Z)$ (where $1 / p+1 / p^{\prime}=1$ ) is weakly compact and the norm functional is weakly lower semicontinuous. Let $A: W_{0}^{1, p}(Z) \mapsto W^{-1, p^{\prime}}(Z)$ be the nonlinear operator defined by

$$
\langle A x, y\rangle \stackrel{d f}{=} \int_{Z}\|\nabla x(z)\|_{\mathbb{R}^{N}}^{p-2}(\nabla x(z), \nabla y(z))_{\mathrm{R}^{N}} d z \quad \forall x, y \in W_{0}^{1, p}(Z) .
$$

Here by $\langle\cdot, \cdot\rangle$ we denote the duality brackets for the pair $\left(W_{0}^{1, p}(Z), W^{-1, p^{\prime}}(Z)\right)$. It is straightforward to check that $A$ is demicontinuous and strongly monotone, hence maximal monotone (see $\mathrm{Hu}$ and Papageorgiou [13, Corollary III.1.35, p.309]). For every $n \geqslant 1$, we have

$$
x_{n}^{*}=A x_{n}-\lambda_{1}\left|x_{n}\right|^{p-2} x_{n}-u_{n}^{*},
$$

where $u_{n}^{*} \in \partial \psi\left(x_{n}\right)$, for $n \geqslant 1$. From Chang [7, Theorem 2.2] and Clarke [8, Theorem 2.7.5, p.83], we know that $u_{n}^{*} \in L^{p^{\prime}}(Z)$ and $u_{n}^{*}(z) \in \partial j\left(z, x_{n}(z)\right)$ almost everywhere on $Z$. 
Because $\left(1+\left\|x_{n}\right\|\right)\left\|x_{n}^{*}\right\|_{*} \rightarrow 0$ as $n \rightarrow+\infty$, so choosing a subsequence if necessary, we have $\left|\left\langle x_{n}^{*}, x_{n}\right\rangle\right| \leqslant 1 / n$. Thus, from (1), we have

$$
-\left\|\nabla x_{n}\right\|_{p}^{p}+\lambda_{1}\left\|x_{n}\right\|_{p}^{p}+\left(u_{n}^{*}, x_{n}\right)_{p p^{\prime}} \leqslant 1 / n
$$

(by $(\cdot, \cdot)_{p p^{\prime}}$ we denote the duality brackets for the pair $\left(L^{p}(Z), L^{p^{\prime}}(Z)\right)$ ). As $\left|p \phi\left(x_{n}\right)\right| \leqslant$ $p M_{1}$ for all $n \geqslant 1$, also

$$
\left\|\nabla x_{n}\right\|_{p}^{p}-\lambda_{1}\left\|x_{n}\right\|_{p}^{p}-\int_{Z} p j\left(z, x_{n}(z)\right) d z \leqslant p M_{1} .
$$

Adding (2) and (3), we obtain

$$
\int_{Z}\left(u_{n}^{*}(z) x_{n}(z)-p j\left(z, x_{n}(z)\right)\right) d z \leqslant \frac{1}{n}+p M_{1} .
$$

By virtue of hypothesis $H(j)$ (iv), we can find $M_{2}=M_{2}(\beta)>0$ such that for almost all $z \in Z$, all $|\zeta| \geqslant M_{2}$ and all $u^{*}(z, \zeta) \in \partial j(z, \zeta)$, we have

$$
u^{*}(z, \zeta) \zeta-p j(z, \zeta)>\frac{\beta}{2}|\zeta|^{\mu}
$$

From the Lebourg mean value theorem (see Lebourg [15] or Clarke [8, Theorem 2.3.7, p.41], we know that for almost all $z \in Z$ and all $\zeta \in \mathbb{R}$, we can find $t \in(0,1)$ and $\eta \in \partial j(z, t \zeta)$, such that

$$
|j(z, \zeta)-j(z, 0)| \leqslant|\eta||\zeta|
$$

and so from hypothesis $H(j)$ (iii), for almost all $z \in Z$ and all $\zeta \in \mathbb{R}$, we have

$$
|j(z, \zeta)| \leqslant|j(z, 0)|+a(z)|\zeta|+c|\zeta|^{p} \leqslant a_{1}(z)\left(1+|\zeta|^{p}\right),
$$

with $a_{1} \in L^{\infty}(Z)$, namely $a_{1}(z) \stackrel{d f}{=}|j(z, 0)|+a(z)+c$ (recall that from $H(j)$ (vi) we have that $j(\cdot, 0) \in L^{\infty}(Z)$ ). Hence and from hypothesis $H(j)$ (iii), for almost all $z \in Z$, all $|\zeta|<M_{2}$ and all $u^{*}(z, \zeta) \in \partial j(z, \zeta)$, we have

$$
\left|u^{*}(z, \zeta) \zeta-p j(z, \zeta)\right| \leqslant a_{2}(z)
$$

with $a_{2} \in L^{\infty}(Z)$, namely $a_{2}(z) \stackrel{d f}{=} M_{2} a(z)+c M_{2}^{p}+p\left(1+M_{2}^{p}\right) a_{1}(z)$. Finally from (5) and (7), we can say that for almost all $z \in Z$, all $\zeta \in \mathbb{R}$ and all $u^{*}(z, \zeta) \in \partial j(z, \zeta)$, we have

$$
\frac{\beta}{2}|\zeta|^{u}-a_{3}(z) \leqslant u^{*}(z, \zeta) \zeta-p j(z, \zeta)
$$


with $a_{3} \in L^{\infty}(Z)$, namely $a_{3}(z) \stackrel{d f}{=} a_{2}(z)+(\beta / 2) M_{2}^{\mu}$. Thus, returning to (4), we have that

$$
\frac{\beta}{2}\left\|x_{n}\right\|_{\mu}^{\mu} \leqslant \int_{Z}\left(u_{n}^{*}(z) x_{n}(z)-p j\left(a, x_{n}(z)\right)\right) d z+\left\|a_{3}\right\|_{1} \leqslant \frac{1}{n}+p M_{1}+\left\|a_{3}\right\|_{\infty}
$$

and so the sequence $\left\{x_{n}\right\}_{n \geqslant 1} \subseteq L^{\mu}(Z)$ is bounded, that is,

$$
\left\|x_{n}\right\|_{\mu} \leqslant c_{1} \quad \forall \geqslant 1
$$

with $c_{1}>0$, namely $c_{1} \stackrel{d f}{=}\left[(2 / \beta)\left(1+p M_{1}+\left\|a_{3}\right\|_{\infty}\right)\right]^{1 / \mu}$.

Let $p^{*}$ be the Sobolev critical exponent, defined by

$$
p^{*} \stackrel{d f}{=} \begin{cases}\frac{N p}{N-p} & \text { if } N>p \\ +\infty & \text { if } N \leqslant p .\end{cases}
$$

Let us choose $q$ such that $p<q<\min \left\{p^{*}, p(\max \{N, p\}+\mu) / \max \{N, p\}\right\}$. From (6), we see that for almost all $z \in Z$ and all $\zeta \in \mathbb{R}$, we have that

$$
j(z, \zeta) \leqslant c_{2}+c_{3}|\zeta|^{q}
$$

with $c_{2} \stackrel{d f}{=} 2\left\|a_{1}\right\|_{\infty}$ and $c_{3} \stackrel{d f}{=}\left\|a_{1}\right\|_{\infty}$. Let

$$
\vartheta \stackrel{d f}{=} \begin{cases}\frac{p^{*}(q-\mu)}{q\left(p^{*}-\mu\right)} & \text { if } N>p, \\ 1-\frac{\mu}{q} & \text { if } N \leqslant p .\end{cases}
$$

Using the interpolation inequality (see for example, Brezis [5, Remarque 2, p.57] and noting that $0<\vartheta<1$ is chosen such that $1 / q$ is the "convex combination" of $1 / \mu$ and $1 / p^{*}$, namely $\left.1 / q=(1-\vartheta) / u+\vartheta / p^{*}\right)$, from inequality (8) and the Sobolev embedding theorem, for $n \geqslant 1$, we have

$$
\left\|x_{n}\right\|_{q} \leqslant\left\|x_{n}\right\|_{\mu}^{1-\theta}\left\|x_{n}\right\|_{p^{*}}^{\vartheta} \leqslant c_{1}^{1-\theta}\left\|x_{n}\right\|_{p^{*}}^{\vartheta} \leqslant c_{4}\left\|x_{n}\right\|^{\ominus}
$$

with some $c_{4}>0$. Recall that for all $n \geqslant 1$, we have that

$$
\frac{1}{p}\left\|\nabla x_{n}\right\|_{p}^{p}-\frac{\lambda_{1}}{p}\left\|x_{n}\right\|_{p}^{p}-\int_{Z} j\left(z, x_{n}(z)\right) d z \leqslant M_{1} .
$$


So using also inequality (9), continuity of the imbedding $L^{q}(Z) \subseteq L^{P}(Z)$, the Young inequality and inequality (10), we have

$$
\begin{aligned}
\frac{1}{p}\left\|\nabla x_{n}\right\|_{p}^{p} & \leqslant \frac{\lambda_{1}}{p}\left\|x_{n}\right\|_{q}^{q}+c_{2}|Z|+c_{3}\left\|x_{n}\right\|_{q}^{p}+M_{1} \\
& \leqslant c_{5}\left\|x_{n}\right\|_{q}^{p}+c_{2}|Z|+c_{3}\left\|x_{n}\right\|_{q}^{q}+M_{1} \\
& \leqslant c_{5}+c_{5}\left\|x_{n}\right\|_{q}^{q}+c_{2}|Z|+c_{3}\left\|x_{n}\right\|_{q}^{q}+M_{1} \\
& =c_{6}+c_{7}\left\|x_{n}\right\|_{q}^{q} \leqslant c_{6}+c_{4}^{q} c_{7}\left\|x_{n}\right\|^{\theta q}
\end{aligned}
$$

where $c_{5} \stackrel{d f}{=} \lambda_{1}|Z|^{(q-p) / q} / p, c_{6} \stackrel{d f}{=} c_{5}+c_{2}|Z|+M_{1}$ and $c_{7} \stackrel{d f}{=} c_{3}+c_{5}$. Using also the Poincaré inequality, we obtain.

$$
\left\|\nabla x_{n}\right\|_{p}^{p} \leqslant c_{8}\left\|\nabla x_{n}\right\|_{p}^{\theta q}+c_{9}
$$

with some $c_{8}=c_{8}\left(\lambda_{1}\right)>0$ and $c_{9} \stackrel{\text { df }}{=} p c_{6}$.

Let us estimate the exponent $\vartheta q$. First suppose $N>p$. Since $N q<N p+\mu p$ (recall the choice of $q$ ), we have that

$$
\vartheta q=\frac{p^{*}(q-\mu)}{p^{*}-\mu}=\frac{N p}{N-p} \cdot \frac{(q-\mu)(N-p)}{N p-N \mu+\mu p}<\frac{N p}{N-p} \cdot \frac{(q-\mu)(N-p)}{N q-N \mu}=p .
$$

Now suppose that $N \leqslant p$. Then from the choice of $q$, we have that

$$
q<\min \left\{p^{*}, p \frac{\max \{N, p\}+\mu}{\max \{N, p\}}\right\}=p \cdot \frac{p+\mu}{p}=p+\mu
$$

and so $q-\mu<p$. Hence we have

$$
\vartheta q=\left(1-\frac{\mu}{q}\right) q=q-\mu<p .
$$

Therefore we see that in both cases, we have that $\vartheta q<p$. Then from (11), it follows that the sequence $\left\{x_{n}\right\}_{n \geqslant 1} \subseteq W_{0}^{1, p}(Z)$ is bounded. Thus by passing to a subsequence if necessary, we may assume that $x_{n} \rightarrow x$ weakly in $W_{0}^{1, p}(Z)$, so also $x_{n} \rightarrow x$ in $L^{p}(Z)$. Thus we have $\left(x_{n}^{*}, x_{n}-x\right) \rightarrow 0$ as $n \rightarrow+\infty$, so from (1), we have

$$
\left\langle A x_{n}, x_{n}-x\right\rangle-\lambda_{1}\left(\left|x_{n}\right|^{p-2} x_{n}, x_{n}-x\right)_{p p^{\prime}}-\left(u_{n}^{*}, x_{n}-x\right)_{p p^{\prime}} \rightarrow 0 \text { as } n \rightarrow+\infty \text {. }
$$

From the continuity of the operator $L^{p}(X) \ni x \mapsto|x|^{p-2} x \in L^{p^{\prime}}(Z)$, we have that $\left|x_{n}\right|^{p-2} x_{n} \rightarrow|x|^{p-2} x$ in $L^{p^{\prime}}(Z)$ as $n \rightarrow+\infty$. As $u_{n}^{*} \in L^{p^{\prime}}(Z)$ and $u_{n}^{*}(z) \in \partial j\left(z, x_{n}(z)\right)$ 
almost everywhere on $Z$, from hypothesis $H(j)$ (iii), we see that the sequence $\left\{u_{n}^{*}\right\}_{n \geqslant 1} \subseteq L^{p^{\prime}}(Z)$ is bounded. Thus, we obtain that

$$
\limsup _{n \rightarrow+\infty}\left\langle A x_{n}, x_{n}-x\right\rangle \leqslant 0 .
$$

Because $A$ is maximal monotone, it is generalised pseudomonotone as well (see Hu and Papageorgiou [13, Definition III.62 and Remark III.6.3, p.365]) and so we have

$$
\left\langle A x_{n}, x_{n}\right\rangle \rightarrow\langle A x, x\rangle \text { as } n \rightarrow+\infty,
$$

thus

$$
\left\|\nabla x_{n}\right\|_{p}^{p} \rightarrow\|\nabla x\|_{p}^{p} \text { as } n \rightarrow+\infty .
$$

On the other hand, since $x_{n} \rightarrow x$ weakly in $W_{0}^{1, p}(Z)$ as $n \rightarrow+\infty$, we have also that $\nabla x_{n} \rightarrow \nabla x$ weakly in $L^{p}\left(Z, \mathbb{R}^{N}\right)$ as $n \rightarrow+\infty$ and since $L^{p}\left(Z, \mathbf{R}^{N}\right)$ is uniformly convex, it has the Kadec-Klee property (see Hu and Papageorgiou [13, Definition I.1.72(d), p.28]). Therefore we conclude that $\nabla x_{n} \rightarrow \nabla x$ in $L^{p}\left(Z, \mathbb{R}^{N}\right)$ as $n \rightarrow+\infty$ and so $x_{n} \rightarrow x$ in $W_{0}^{1, p}(Z)$ as $n \rightarrow+\infty$. Thus $\phi$ satisfies the nonsmooth Cerami condition.

Lemma 3. If hypotheses $H(j)$ hold then there exists $r_{0}>0$ such that for all $0<r<r_{0}$, we have $\inf \{\phi(x):\|x\|=r\}>0$.

ProOF: By virtue of hypothesis $H(j)(v)$, we can find $\delta>0$ such that for almost all $z \in Z$ and all $\zeta$ such that $|\zeta| \leqslant \delta$, we have

$$
j(z, \zeta) \leqslant-\frac{\lambda_{1}}{2 p}|\zeta|^{p}
$$

On the other hand, from the proof of Lemma 2 (see inequality (9)) we see that for almost all $z \in Z$ and all $\zeta$ such that $|\zeta|>\delta$, we have

$$
|j(z, \zeta)| \leqslant c_{10}|\zeta|^{q}
$$

with $c_{10} \stackrel{d f}{=} c_{2} \delta^{-q}+c_{3}$ and with $p<q<p^{*}$. From (12) and (13), it follows that for almost all $z \in Z$ and all $\zeta \in \mathbb{R}$, we have

$$
j(z, \zeta) \leqslant-\frac{\lambda_{1}}{2 p}|\zeta|^{p}+c_{11}|\zeta|^{q}
$$

where $c_{11} \stackrel{d f}{=} c_{10}+\lambda_{1} /\left(2 p \delta^{q-p}\right)$. Hence, for every $x \in W_{0}^{1, p}(Z)$, we have

$$
\begin{aligned}
\phi(x) & \geqslant \frac{1}{p}\|\nabla x\|_{p}^{p}-\frac{\lambda_{1}}{p}\|x\|_{p}^{p}+\frac{\lambda_{1}}{2 p}\|x\|_{p}^{p}-c_{11}\|x\|_{q}^{q} \\
& =\frac{1}{p}\left\|\nabla_{x}\right\|_{p}^{p}-\frac{\lambda_{1}}{2 p}\|x\|_{p}^{p}-c_{11}\|x\|_{q}^{q} .
\end{aligned}
$$


Now, using the Rayleigh quotient, the Sobolev embedding theorem and the Poincare inequality, we obtain

$$
\begin{aligned}
\phi(x) & \geqslant \frac{1}{p}\|\nabla x\|_{p}^{p}-\frac{1}{2 p}\|\nabla x\|_{p}^{p}-c_{11}\|x\|^{q} \\
& =\frac{1}{2 p}\|\nabla x\|_{p}^{p}-c_{11}\|x\|^{q} \geqslant c_{12}\|x\|^{p}-c_{11}\|x\|^{q},
\end{aligned}
$$

with some $c_{12} \geqslant 0$. Since $p<q$, from the last inequality, we see that choosing $0<r_{0}<$ $\left(c_{12} / c_{11}\right)^{1 /(q-p)}$ we shall have that $\inf \{\phi(x):\|x\|=r\}>0$ for any $0<r<r_{0}$.

Now are ready to state and prove an existence result for (HVI).

THEOREM 4. If hypotheses $H(j)$ hold, then the problem (HVI) has a nontrivial solution $x_{0} \in W_{0}^{1, p}(Z)$.

Proof: From hypothesis $H(j)$ (vi), we have that $\phi(0) \leqslant 0$ and $\phi\left(\xi u_{1}\right) \leqslant 0$. From Proposition 2, we know that $\phi$ satisfies the nonsmooth Cerami condition and from Propsition 3, we can choose $r<\min \left\{r_{0}, \xi\right\}$ such that $\inf \{\phi(x):\|x\|=r\}>0$. Now we can apply Theorem 1 (with $x_{1}=0, x_{2}=\xi u_{1}$ and $r$ defined above) and produce $x_{0} \in W_{0}^{1, p}(Z)$ such that $0 \in \partial \phi\left(x_{0}\right)$ and $\phi\left(x_{0}\right) \geqslant \inf \{\phi(x):\|x\|=r\}>0$. Clearly $x_{0} \neq 0$ and we have

$$
A x_{0}=\lambda_{1}\left|x_{0}\right|^{p-2} x_{0}+u^{*} \quad \text { in } W^{-1, q}(Z),
$$

with $u^{*} \in \partial \psi\left(x_{0}\right)$, hence $u^{*} \in L^{p^{\prime}}(Z)$ and $u^{*}(z) \in \partial j\left(z, x_{0}(z)\right)$ almost everywhere on $Z$. Let $\varphi \in C_{0}^{\infty}(Z)$. We have

$$
\left\langle A x_{0}, \varphi\right\rangle=\lambda_{1}\left(\left|x_{0}\right|^{p-2} x_{0}, \varphi\right)_{p p^{\prime}}+\left(u^{*}, \varphi\right)_{p p^{\prime}}
$$

and by Green's theorem

$$
\left\langle-\operatorname{div}\left(\left\|\nabla x_{0}\right\|^{p-2} \nabla x_{0}\right), \varphi\right\rangle=\lambda_{1}\left(\left|x_{0}\right|^{p-2} x_{0}, \varphi\right)_{p p^{\prime}}+\left(u^{*}, \varphi\right)_{p p^{\prime}}
$$

Note that from the representation theorem for the elements in the dual space $W^{-1, p^{\prime}}(Z)=$ $\left(W_{0}^{1, p}(Z)\right)^{*}$ (see Adams [1, Theorem 3.10, p.50]), we have that $\operatorname{div}\left(\left\|\nabla x_{0}\right\|^{p-2} \nabla x_{0}\right) \in$ $W^{-1, p^{\prime}}(Z)$. Since $C_{0}^{\infty}(Z)$ is dense in $W_{0}^{1, p}(Z)$, we deduce that

$$
\left\{\begin{array}{l}
-\operatorname{div}\left(\left\|\nabla x_{0}(z)\right\|^{p-2} \nabla x_{0}(z)\right)-\lambda_{1}\left|x_{0}(z)\right|^{p-2} x_{0}(z) \\
\quad=u^{*}(z) \in \partial j\left(z, x_{0}(z)\right) \text { almost everywhere on } Z \\
\left.x_{0}\right|_{\Gamma}=0,
\end{array}\right.
$$

and so $x_{0}$ is a nontrivial solution of (HVI). 
REMARK 5. From the proof of Theorem 4 one can see that the part of hypothesis $H(j)$ (vi) concerning the existence of $\xi \neq 0$ such that $\int_{Z} j\left(z, \xi u_{1}(z)\right) d z \geqslant 0$ can be replaced by a more general one, not involving the first eigenfunction $u_{1}$, namely:

There exists $\bar{x} \in W_{0}^{1, p}(Z), \bar{x} \neq 0$, such that

$$
\frac{p \int_{z} j(z, \bar{x}(z)) d z}{\|\bar{x}\|_{p}^{p}} \geqslant \frac{\|\nabla \bar{x}\|_{p}^{p}}{\|\bar{x}\|_{p}^{p}}-\lambda_{1} .
$$

As a simple illustrative example along the lines of those by Panagiotopoulos [17], we consider the problem

$$
\left\{\begin{array}{l}
-\operatorname{div}\left(\|\nabla x(z)\|^{p-2} \nabla x(z)\right)-\lambda_{1}|x(z)|^{p-2} x(z) \\
\quad=f(x(z)) \text { almost everywhere on } Z \\
\left.x\right|_{\Gamma}=0 .
\end{array}\right.
$$

where $2<p<+\infty$ and $f: \mathbb{R} \mapsto \mathbb{R}$ is defined by

$$
f(\zeta)= \begin{cases}2 \zeta+2 & \text { if } \zeta \leqslant-1 \\ s|\zeta|^{s-1} & \text { if }-1<\zeta<0 \\ -s \zeta^{s-1} & \text { if } 0 \leqslant \zeta<1 \\ 2 \zeta-2 & \text { if } 1 \leqslant \zeta\end{cases}
$$

with $1<s<p$ (see Figure 1). Let $j: \mathbb{R} \mapsto \mathbb{R}$ be defined by $j(\zeta) \stackrel{d f}{=} \int_{0}^{\zeta} f(\xi) d \xi$ (see Figure 2). Then

$$
j(\zeta)= \begin{cases}-|\zeta|^{s} & \text { if }|\zeta| \leqslant 1 \\ \left(\zeta^{2}-2|\zeta|\right) & \text { if }|\zeta|>1\end{cases}
$$

Since $f$ is discontinuous at $\zeta= \pm 1$, the problem need not have a solution (see Stuart [19]). Following Chang [7], in order to obtain a solution, we pass to a multivalued version of (14), by filling in the gaps at the discontinuity points. So we introduce

$$
\hat{f}(\zeta)= \begin{cases}f(\zeta) & \text { if } \zeta \neq+1 \\ {[0, s]} & \text { if } \zeta=-1 \\ {[-s, 0]} & \text { if } \zeta=1\end{cases}
$$

From Clarke [8, p.34], we know that $j$ is locally Lipschitz and $\partial j(\zeta)=\hat{f}(\zeta)$ for all $\zeta \in \mathbb{R}$ (see Figure 3). So instead of (14), we consider

$$
\left\{\begin{array}{l}
\operatorname{div}\left(\|\nabla x(z)\|^{p-2} \nabla x(z)\right)-\lambda_{1}|x(z)|^{p-2} x(z) \\
\quad \in \partial j(x(z)) \text { almost everywhere on } Z \\
\left.x\right|_{\Gamma}=0 .
\end{array}\right.
$$


We can easily check that hypotheses $H(j)$ are satisfied and we can apply Theorem 4 to obtain a nontrivial solution $x \in W_{0}^{1, p}(Z)$ for problem (16).

We can have another example, by replacing function $f$ in (14) (the right hand side in problem (14)) by the function $g: Z \times \mathbb{R} \mapsto \mathbb{R}$ defined by $g(z, \zeta)=b(z) f(\zeta)$, where $b \in L^{\infty}(Z)$, with $b(z) \geqslant \gamma>0$ for almost all $z \in Z$ and $f: \mathbf{R} \mapsto \mathbf{R}$ defined by (15). Then also hypotheses $H(j)$ are satisfed and from Theorem 4 we obtain a nontrivial solution $x \in W_{0}^{1, p}(Z)$ for problem (16).
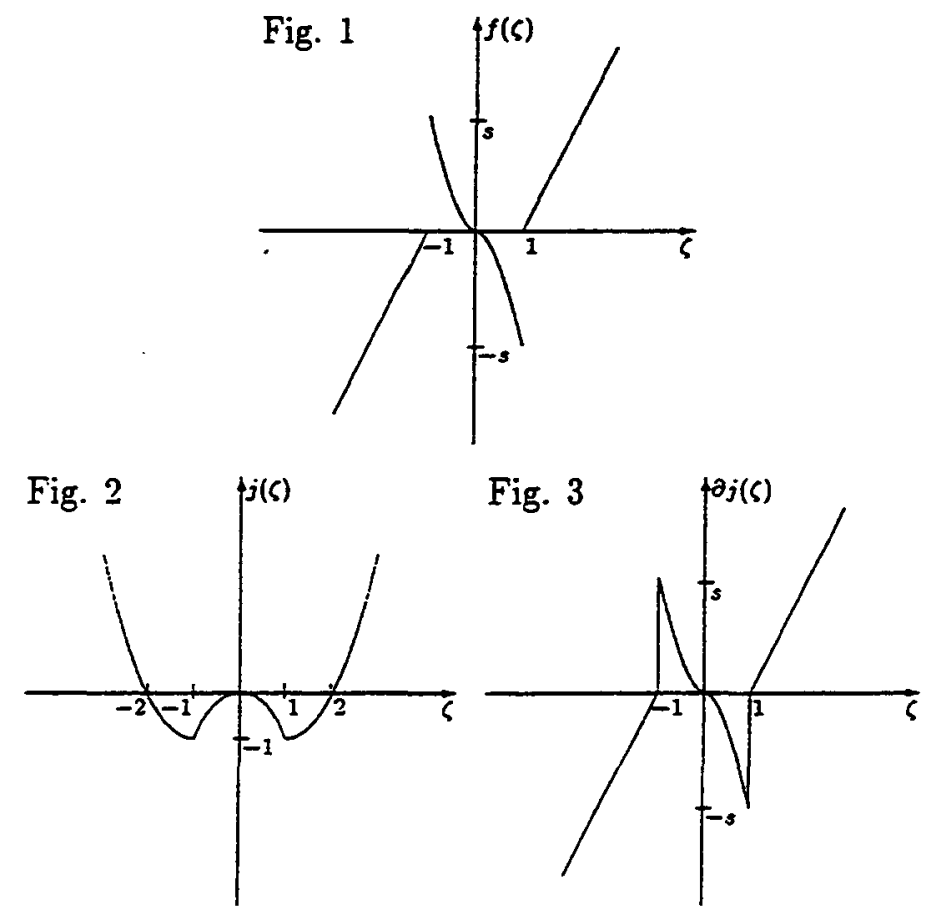

\section{REFERENCES}

[1] R. Adams, Sobolev spaces (Academic Press, New York, 1975).

[2] S. Ahmad, A. Lazer and J. Paul, 'Elementary critical point theory and perturbations of elliptic boundary value problems at resonance', Indiana Univ. Math. J. 25 (1976), 933-944.

[3] A. Ambrosetti and P.H. Rabinowitz, 'Dual variational methods in critical point theory and applications', J. Funct. Anal. 14 (1973), 349-381.

[4] P. Bartolo, V. Benci and D. Fortunato, 'Abstract critical point theorems and applications to some nonlinear problems with "strong" resonance at infinity', Nonlinear Anal. 7 (1983), 981-1012.

[5] H. Brezis, Analyse fonctionnelle. Théorie et applications (Masson, Paris, 1983).

[6] G. Cerami, 'An existence criterion for the critical points on unbounded manifolds', (in 
Italian), Instit. Lombardo Accad. Sci. Lett. Rend. A 112 (1978), 332-336.

[7] K.C. Chang, 'Variational methods for nondifferentiable functionals and their applications to partial differential equations', J. Math. Anal. Appl. 80 (1981), 102-129.

[8] F.H. Clarke, Optimization and nonsmooth analysis (Wiley, New York, 1983).

[9] L. Gasiński and N.S. Papageorgiou, 'Nonlinear hemivariational inequalities at resonance', Bull. Austral. Math. Soc. 60 (1999), 353-364.

[10] L. Gasiński and N.S. Papageorgiou, 'Existence of solutions and of multiple solutions for eigenvalue problems of hemivariational inequalities', Adv. Math. Sci. Appl. (to appear).

[11] D. Goeleven, D. Motreanu and P.D. Panagiotopoulos, 'Multiple solutions for a class of eigenvalue problems in hemivariational inequalities', Nonlinear Anal. 29 (1997), 9-26.

[12] D. Goeleven, D. Motreanu and P.D. Panagiotopoulos, 'Eigenvalue problems for variational-hemivariational inequalities at resonance', Nonlinear Anal. 33 (1998), 161-180.

[13] S. Hu and N.S. Papageorgiou, Handbook of multivalued analysis. Volume I: Theory (Kluwer, Dordrecht, The Netherlands, 1997).

[14] N. Kourogenis and N.S. Papageorgiou, 'Nonsmooth critical point theory and applications', Kodai Math. J. 23 (2000), 108-135.

[15] G. Lebourg, 'Valeur mayenne pour gradient généralisé', C. R. Acad. Sci. Paris Ser. A-B 281 (1975), 795-797.

[16] P. Lindqvist, 'On the equation $\operatorname{div}\left(\|\nabla x\|^{p-2} \nabla x\right)+\lambda|z|^{p-2} x=0$ ', Proc. Amer. Math. Soc. 109 (1990), 157-164.

[17] P.D. Panagiotopoulos, Hemivariational inequalities. Applications to mechanics and engineering (Springer-Verlag, Berlin, Heidelberg, New York, 1993).

[18] P.H. Rabinowitz, Minimax methods in critical point theory with applications to differential equations, CBMS, Regional Conference Series in Math. 65 (American Mathematical Society, Providence, R.I., 1986).

[19] C. Stuart, 'Differential equations with discontinuous nonlinearities', Arch. Rational Mech. Anal. 63 (1976), 59-75.

[20] K. Thews, 'Nontrivial solutions of elliptic equations at resonance', Proc. Roy. Soc. Edinburgh Ser. A 85 (1980), 119-129.

[21] P. Tolksdorf, 'Regularity for a more general class of quasilinear elliptic equations', $J$. Differential Equations 51 (1984), 126-150.

[22] J. Ward, 'Applications of critical point theory to weakly nonlinear boundary value problems at resonance', Houston J. Math. 10 (1984), 291-305.

[23] C.K. Zhong, 'On Ekeland's variational principle and a minimax theorem', J. Math. Anal. Appl. 205 (1997), 239-250.

Jagellonian University

Institute of Computer Science

ul. Nawojki 11

30072 Cracow

Poland
National Technical University

Department of Mathematics

Zografou Campus

Athens 15780

Greece 